\title{
Analysis of gastrointestinal metastasis of primary lung cancer: Clinical characteristics and prognosis
}

\author{
NAOHIRO TAIRA ${ }^{1}$, TSUTOMU KAWABATA ${ }^{1}$, ATSUSHI GABE ${ }^{2}$, TOMONORI FURUGEN ${ }^{1}$, TAKAHARU ICHI ${ }^{1}$, \\ KAZUAKI KUSHI $^{1}$, TOMOFUMI YOHENA ${ }^{1}$, HIDENORI KAWASAKI ${ }^{1}$, DAISUKE HIGUCHI ${ }^{3}$, KENJI CHIBANA ${ }^{4}$, \\ KAORI FUJITA ${ }^{4}$, ATSUSHI NAKAMOTO ${ }^{4}$, ISOKO OWAN ${ }^{4}$, MUTSUO KUBA $^{4}$ and KIYOSHI ISHIKAWA ${ }^{1}$ \\ ${ }^{1}$ Department of General Surgery, National Hospital Organization, Okinawa National Hospital, Ginowan, Okinawa 9012214; \\ ${ }^{2}$ Department of General Surgery, Okinawa Prefectural Nanbu Medical Center and Children's Medical Center, Haebaru, \\ Okinawa 9011193; Departments of ${ }^{3}$ Gastroenterology and ${ }^{4}$ Respiratory Medicine, National Hospital Organization, \\ Okinawa National Hospital, Ginowan, Okinawa 9012214, Japan
}

Received September 21, 2015; Accepted March 3, 2017

DOI: $10.3892 / 01.2017 .6382$

\begin{abstract}
The prevalence of gastrointestinal metastasis of lung cancer is low. The aim of the present study was to analyze the frequency and clinical characteristics of metastases to the gastrointestinal tract by retrospectively assessing the clinical records of 2,066 patients with lung cancer. A total of 7 patients $(0.33 \%)$ were diagnosed with gastrointestinal metastasis, including 4 patients with adenocarcinoma, 1 patient with large cell carcinoma and 2 patients with pleomorphic carcinoma. Furthermore, 3 of the patients presented with small bowel metastases, 2 with gastric metastases, 1 with large bowel metastasis and 1 with metastasis of the appendix. The mean time between the diagnosis of the lung tumors and the identification of gastrointestinal metastasis was 13.5 months (range, 3-49 months). The mean time between the identification of the gastrointestinal metastasis and mortality was 100.6 days (range, 21-145 days). In conclusion, the prognosis of patients with recurrence in distant organs, including the gastrointestinal tract, may be worse than patients with recurrence in distant organs, excluding the gastrointestinal tract, particularly those with symptomatic gastrointestinal metastasis. Therefore, the presence of clinical gastrointestinal metastasis may be life threatening; comprehensive evaluations are required to detect and monitor gastrointestinal metastasis during follow-up.
\end{abstract}

\section{Introduction}

Primary lung cancer frequently metastasizes to the brain, liver, adrenal glands and bones $(1,2)$. However, the clinical incidence

Correspondence to: Dr Naohiro Taira, Department of General Surgery, National Hospital Organization, Okinawa National Hospital, Ganeko 3-20-14, Ginowan, Okinawa 9012214, Japan E-mail: naohiro_taira@yahoo.co.jp

Key words: lung cancer, gastrointestinal metastasis, analysis, characteristics, prognosis of gastrointestinal metastasis of lung cancer has been reported to be as low as $0.2-1.7 \%$ (3-8). By contrast, the rate of metastasis of primary lung cancer to the gastrointestinal tract in autopsy studies is higher than the clinical frequency of gastrointestinal metastasis. The cause of mortality for lung cancer patients is not frequently gastrointestinal metastasis $(5,9,10)$ and life-threatening gastrointestinal metastases occur only rarely (5). The low clinical incidence of gastrointestinal metastasis may be because the metastasis is usually asymptomatic. As the clinical incidence and associated mortality rate are low and the lack of symptoms may cause studies to be difficult to organize, the understanding of the clinical presentation of metastases of primary lung cancer to the gastrointestinal tract is currently incomplete.

The aim of the present study was to analyze the frequency and clinical characteristics of metastasis of primary lung cancer to the gastrointestinal tract by retrospectively assessing the clinical records of patients with primary lung cancer to identify the individuals diagnosed with metastasis to the gastrointestinal tract, and subsequently analyzing the clinical data of those individuals.

\section{Patients and methods}

Patients. The clinical records of all patients with a diagnosis of lung cancer treated at the National Hospital Organization, Okinawa National Hospital (Ginowan, Okinawa, Japan) between January 2004 and December 2014 were retrospectively reviewed. All patients with pathological evidence of gastrointestinal metastasis of primary lung cancer were included.

Patient characteristics, including sex, age, histological type, Tumor-Node-Metastasis classification according to the 7th edition of the Lung Cancer Stage Classification system (11), location of the primary lung cancer, location of the gastrointestinal metastases, clinical presentations, diagnostic procedures, other metastatic sites locations at the time of gastrointestinal metastasis, interval between the diagnosis of the lung tumors and the diagnosis of gastrointestinal metastasis, and survival history, were investigated. 


\section{Results}

Characteristics of the patients with gastrointestinal metastasis. A total of 2,066 lung cancer patients were diagnosed or referred to the National Hospital Organization, Okinawa National Hospital, between January 2004 and December 2014 , of whom 7 patients $(0.33 \%)$ were diagnosed with gastrointestinal metastasis.

The characteristics of the patients with gastrointestinal metastasis are listed in Table I. All patients were male. The median age was 66 years (range, 57-71 years) at the time of the diagnosis of gastrointestinal metastasis. In total, 4 patients had adenocarcinoma, 1 patient had large cell carcinoma and 2 patients had pleomorphic carcinoma. Furthermore, 3 patients were in stage IA, 1 patient was in stage IB, 1 patient was in stage IIIA and 2 patients were in stage IVb. Although the clinical stage in case 3 was determined to be T2aN2M0 stage IIIA, surgery was performed after induction chemotherapy, and the postoperative pathological stage was downgraded to T2N0M0, stage IB. The primary location of lung cancer was in the left upper lobe in 5 patients, the right upper lobe in 1 patient and the right lower lobe in 1 patient. The 4 patients with stage IA and IB disease underwent gastroendoscopy and colonoscopy as screening procedures at the time of the diagnosis of lung cancer, and there were no signs of malignancy in any of these cases.

The symptoms, causes of the symptoms, diagnostic tools, systemic treatment after the diagnosis of gastrointestinal metastasis, time between the diagnosis of lung cancer and gastrointestinal metastasis, other metastatic sites, time between the identification of gastrointestinal metastasis and mortality, and the cause of mortality are listed in Table II. Of the 7 patients, 3 exhibited small bowel metastasis, 2 exhibited gastric metastasis, 1 exhibited large bowel metastasis and 1 exhibited metastasis of the appendix. The mean time between the diagnosis of the lung tumors and the identification of the gastrointestinal metastasis was 13.5 months (range, 3-49 months), and the mean time between the identification of gastrointestinal metastasis and mortality was 100.6 days (range, 21-145 days).

Gastric metastasis. Gastroendoscopy was performed in 2 patients due to symptoms of tarry stools and anemia. The gastroendoscopic findings in each case revealed a submucosal tumor (Fig. 1) and irregular gastric ulcers. The 2 patients with gastric metastasis refused surgical resection for palliation, as they were concerned about the potential for a prolonged recovery. In addition, chemotherapy was discontinued, as their performance status worsened despite the chemotherapy. Uncontrolled anemia from chronic bleeding was the cause of mortality.

Small bowel metastasis. In total, 2 out of the 3 patients with small bowel metastasis demonstrated perforation, 1 of who experienced intussusception. The 2 patients were diagnosed by emergency laparotomy, and the intraoperative findings showed multiple metastatic nodules in the mesentery. The patients underwent a partial resection of the small bowel. Chemotherapy was administered to 1 patient after the surgery, but not to the other, due to a worsened performance status after surgery. The cause of mortality was carcinomatous peritonitis, and these patients experienced relatively shorter survival times than the patients with other gastrointestinal metastatic sites.

Large bowel metastasis. The patient with large bowel metastasis underwent colonoscopy due to an elevated serum tumor marker level and increased [18]-fluorine fluorodeoxyglucose (FDG) uptake in the ascending colon upon positron emission tomography-computed tomography (PET-CT). The patient exhibited no abdominal symptoms. Colonoscopy revealed a large protruding tumor in the ascending colon (Fig. 2), which was suspected to be a malignant lesion based on the imaging findings, although the mass was a submucosal tumor and the histological examination of a biopsy specimen obtained from the tissue revealed no malignancy. The patient underwent a right hemicolectomy, as there were no other signs of distant metastasis. The final results of the pathological examination showed a diagnosis of metastatic carcinoma of lung cancer. Liver metastasis appeared 21 months after the surgery, and the patient underwent resection of the metastasis. The patient is currently alive without disease 8 years after the hemicolectomy procedure.

Metastasis to the appendix. The patient with appendiceal metastasis underwent an appendectomy. The appendix was perforated based on the intraoperative findings, and the final results of the pathological examination showed metastatic carcinoma of lung cancer. The metastatic tumor cells had infiltrated all layers of the wall of the appendix. Although chemotherapy was administered after the surgery, the patient succumbed to carcinomatous peritonitis.

\section{Discussion}

According to several autopsy studies, gastrointestinal metastasis of primary lung cancer occurs in $4.7-14.0 \%$ of cases (3-5). However, in past clinical studies, the incidence of gastrointestinal metastasis has been reported to be as low as $0.2-1.7 \%$ (5-8), and in the current study, the clinical prevalence of gastrointestinal metastasis of lung cancer was $\sim 0.33 \%$ $(7 / 2,066)$. The method underlying the spread of metastasis to the intra-abdominal region is believed to involve hematogenous and lymphatic routes (12).

In the present study, the gastrointestinal metastases in 2 cases were formed as submucosal tumors with a normal overlying mucosa. In such cases, metastatic tumors appear in submucosal locations in the gastrointestinal tract with normal overlying mucosa as a result of lymphatic or bloodstream dissemination to the gastrointestinal tract (13). Therefore, it may be difficult to obtain an accurate diagnosis using a biopsy due to the presence of the overlying mucosa.

The most common histological type in the current study was adenocarcinoma. Although certain clinical studies and autopsy series (14-16) have shown adenocarcinoma to be prominent, as observed in the present study, other clinical studies $(3,4)$ have demonstrated that squamous cell carcinoma, large cell carcinoma and pleomorphic carcinoma are more frequent $(5,17-21)$. Therefore, the histological type predominantly associated with gastrointestinal metastasis remains unclear. 
Table I. Characteristics of patients with gastrointestinal metastasis from lung cancer.

\begin{tabular}{|c|c|c|c|c|c|c|}
\hline Case number & Sex & Age, years & Histological type & TNM classification & Stage & Primary lobe \\
\hline 1 & Male & 57 & Adenocarcinoma & T2N0M0 & IB & Right upper lobe \\
\hline 2 & Male & 64 & Adenocarcinoma & $\mathrm{T} 2 \mathrm{~N} 2 \mathrm{M} 1 \mathrm{~b}$ & $\mathrm{IVb}$ & Left upper lobe \\
\hline 3 & Male & 64 & Large cell carcinoma & T1bN0M0 & IA & Left upper lobe \\
\hline 4 & Male & 66 & Pleomorphic carcinoma & T1bN0M0 & IA & Left upper lobe \\
\hline 5 & Male & 67 & Adenocarcinoma & T2N2M0 & IIIA & Left upper lobe \\
\hline 6 & Male & 71 & Pleomorphic carcinoma & T1aN0M0 & IA & Left upper lobe \\
\hline 7 & Male & 71 & Adenocarcinoma & T2N3M1b & $\mathrm{IVb}$ & Right lower lobe \\
\hline
\end{tabular}

TNM, tumor-node-metastasis.

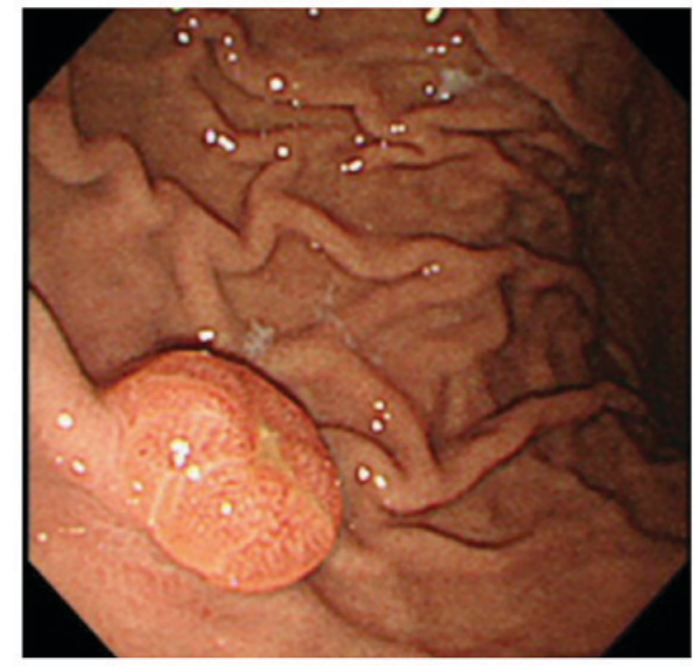

Figure 1. Gastroscopy image showing a gastric submucosal tumor with central erosion in the greater curvature of the stomach.

The left upper lobe was the predominant primary location of lung cancer in the present study. In a study by Yang et al (22), the left and right upper lobes were predominant. By contrast, there were no significant differences in the primary site of lung cancer in an autopsy study by Yoshimoto et al (5). Furthermore, in past case studies $(16,19-21,23)$, the primary site of lung cancer included a range of lobes. To the best of our knowledge, no previous studies have clarified the predominant primary lobe. In general, lung cancer commonly affects the upper lobes more frequently than the lower lobes (24) and the right lung more often than the left (25). Therefore, the primary lobe associated with gastrointestinal metastasis may more frequently be the upper lobes than the lower lobes.

Gastric metastasis arising from lung cancer is extremely rare, and only a few studies have been published on the subject (26-28). In the current study, the 2 patients with gastric metastasis presented with symptoms of tarry stools and anemia due to chronic bleeding, and the cause of mortality was uncontrolled bleeding in each case. Surgical resection of gastric metastasis for palliation may result in prolonged survival. Regarding chemotherapy, chemotherapy-induced gastric perforation as a complication of lung cancer treatment has been reported in a previous study. According to

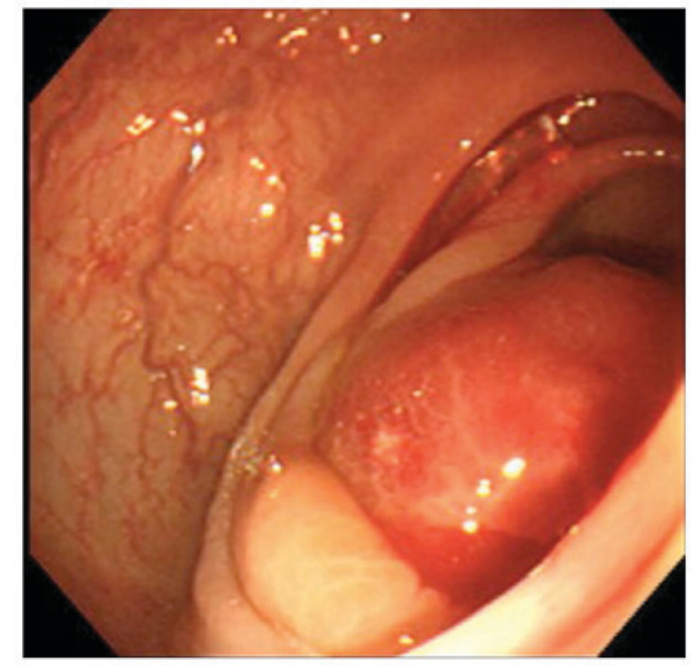

Figure 2. Colonoscopy image showing a protruding submucosal tumor in the ascending colon.

this study (27), chemotherapy-induced necrosis of metastatic tumors may lead to gastric perforation. In the present cases, the administration of chemotherapy may have worsened the bleeding of the gastric metastasis due to necrosis. Therefore, we suggest that surgical resection of gastric metastasis is a beneficial option for palliation in correctly selected patients.

Previous studies have also reported that the small bowel is the most common gastrointestinal metastatic site of lung cancer $(5,22,29)$. The prognosis of such lesions is worse than that of other locations $(22,29)$, and emergent intervention is often required, as small bowel involvement regularly leads to perforation, which is the most common presentation, as well as obstruction or bleeding $(22,29,30)$. In the current study, 3 out of the 7 patients presented with small bowel involvement. Although all of these patients underwent emergent surgery, they exhibited a relatively shorter survival time than the subjects with other gastrointestinal metastatic sites. This may be since small bowel metastasis is typically associated with widespread disease. Stenbygaard et al reported that gastrointestinal metastases usually occur as a component of otherwise widespread metastatic diseases (14). In addition, within the study by McNeill et al, it was reported that 46 patients with small bowel metastases had at least one other site of metastatic 


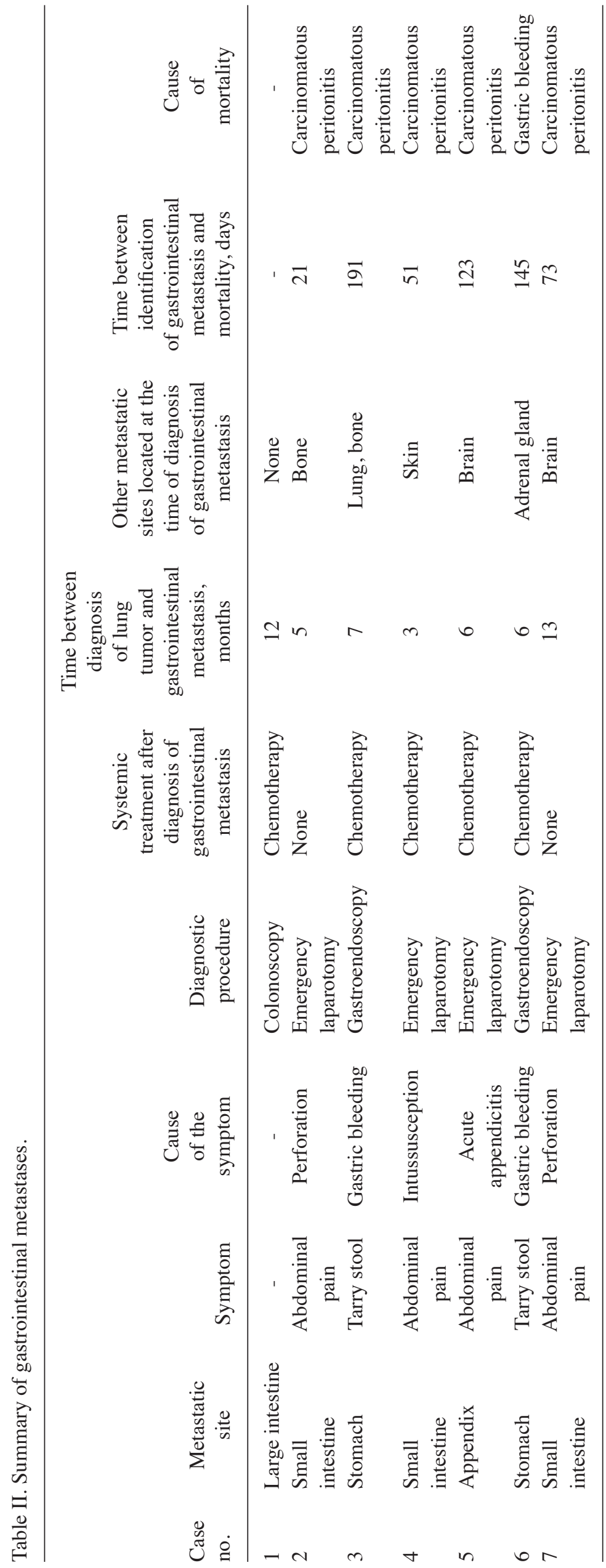


disease, with a mean of 4.8 sites (3). In the present study, multiple metastatic nodules were detected in the mesentery based on the intraoperative findings, and the cause of mortality was carcinomatous peritonitis in each case.

Large bowel metastasis of lung cancer is also extremely rare. In the current study, while 1 patient with metastasis of the appendix developed acute appendicitis and succumbed as a result of carcinomatous peritonitis 4 months later, another patient with metastasis of the ascending colon remains alive 8 years after a right hemicolectomy. In the case of metastasis of the appendix, perforation of the appendix may have led to dissemination to the intra-abdominal region or the metastasis may have been a result of widespread intra-abdominal dissemination with respect to the cause of mortality due to carcinomatous peritonitis.

To the best of our knowledge, only a few studies of long-term survival in cases of large bowel metastasis of lung cancer have been published $(17,31)$. In the current study, we hypothesize that the long-term survival in 1 case was due to the presence of solitary metastasis with resectable lesions.

There was no case of esophageal metastasis in the present study. Esophageal metastasis is also extremely rare, with only a few published clinical studies on lesions of esophageal metastasis $(32,33)$, although the incidence of this condition is $6.4 \%$ according to an autopsy report by Antler et al (4). Therefore, more thorough scientific documentation is required to discuss the characteristics of esophageal metastasis.

The interval period between the diagnosis of the lung tumors and the detection of gastrointestinal metastasis was within 1 year in the present study, with the exception of the patient with metastasis to the appendix. It is noteworthy that the interval in all patients, including those with stage I disease who underwent complete resection, was so short ( $\leq 7$ months). According to the study by Yoshino et al (34), even patients with recurrence in distant organs can expect a long survival time if they receive treatment in the early pathological stage of primary cancer or have resectable recurrent disease. However, there were no cases of gastrointestinal metastasis in this study. The prognosis of patients with recurrence in distant organs, including the gastrointestinal tract, may be worse than that of patients with recurrence in distant organs, excluding the gastrointestinal tract, particularly those with symptomatic gastrointestinal metastasis (22), despite being in the early pathological stage. However, as aforementioned, long-term survival is possible if the gastrointestinal lesions are solitary and resectable. Therefore, physicians should perform comprehensive evaluations to assess asymptomatic gastrointestinal metastasis during follow-up. In the current case of large bowel solitary metastasis, FDG-PET images were effective for detecting the metastases. According to the findings of the study by Lardinois et al, solitary extrapulmonary lesions are observed on PET-CT imaging in $21 \%$ of patients with non-small cell lung cancer (35). Furthermore, PET scanning may reveal a higher incidence of gastrointestinal metastasis than previously suspected (36), although additional studies are required to identify better methods for recognizing and treating gastrointestinal metastasis.

In conclusion, the presence of clinical gastrointestinal metastasis may be life threatening, and comprehensive evaluations are required to detect and monitor gastrointestinal metastasis during follow-up. The limitations of the study were that it was a single center, retrospective study with a small sample size. Therefore, prospective, randomized trials are required to verify the frequency and clinical characteristics of metastases to the gastrointestinal tract.

\section{References}

1. Auerbach O, Garfinkel L and Parks VR: Histologic type of lung cancer in relation to smoking habits, year of diagnosis and sites of metastases. Chest 67: 382-387, 1975.

2. Hillers TK, Sauve MD and Guyatt GH: Analysis of published studies on the detection of extrathoracic metastases in patients presumed to have operable non-small cell lung cancer. Thorax 49: 14-19, 1994.

3. McNeill PM, Wagman LD and Neifeld JP: Small bowel metastases from primary carcinoma of the lung. Cancer 59: 1486-1489, 1987.

4. Antler AS, Ough Y, Pitchumoni CS, Davidian M and Thelmo W: Gastrointestinal metastases from malignant tumors of the lung. Cancer 49: 170-172, 1982.

5. Yoshimoto A, Kasahara K and Kawashima A: Gastrointestinal metastases from primary lung cancer. Eur J Cancer 42: 3157-3160, 2006.

6. Kim SY, Ha HK, Park SW, Kang J, Kim KW, Lee SS, Park SH and Kim AY: Gastrointestinal metastasis from primary lung cancer: CT findings and clinicopathologic features. AJR Am J Roentgenol 193: W197-W201, 2009.

7. Gitt SM, Flint P, Fredell CH and Schmitz GL: Bowel perforation due to metastatic lung cancer. J Surg Oncol 51: 287-291, 1992.

8. Lee PC, Lo C, Lin MT, Liang JT and Lin BR: Role of surgical intervention in managing gastrointestinal metastases from lung cancer. World J Gastroenterol 17: 4314-4320, 2011.

9. Ogata R, Tanio Y, Takashima J, Kato Y, Arizumi T, Takada R, Tabata Y, Shimazu K and Fushimi H: Retrospective analysis of immediate cause of death in lung cancer-two case reports of lung cancer deaths due to bowel necrosis. Gan To Kagaku Ryoho 38: 987-990, 2011 (In Japanese).

10. Nichols L, Saunders R and Knollmann FD: Causes of death of patients with lung cancer. Arch Pathol Lab Med 136: 1552-1557, 2012.

11. Goldstraw P, Crowley J, Chansky K, Giroux DJ, Groome PA, Rami-Porta R, Postmus PE, Rusch V and Sobin L; International Association for the Study of Lung Cancer International Staging Committee; Participating Institutions: The IASLC lung cancer staging project: Proposals for the revision of the TNM stage groupings in the forthcoming (seventh) edition of the TNM classification of malignant tumors. J Thorac Oncol 2: 706-714, 2007.

12. Leidich RB and Rudolph LE: Small bowel perforation secondary to metastatic lung carcinoma. Ann Surg 193: 67-69, 1981.

13. Simchuk EJ and Low DE: Direct esophageal metastasis from a distant primary tumor is a submucosal process: A review of six cases. Dis Esophagus 14: 247-250, 2001

14. Stenbygaard LE and Sorensen JB: Small bowel metastases in non-small cell lung cancer. Lung Cancer 26: 95-101, 1999.

15. Stenbygaard LE and Sørensen JB: Small bowel metastases in non-small cell lung cancer. Lung Cancer 26: 95-101, 1999.

16. Okazaki R, Ohtani H, Takeda K, Sumikawa T, Yamasaki A, Matsumoto S and Shimizu E: Gastric metastasis by primary lung adenocarcinoma. World J Gastrointest Oncol 2: 395-398, 2010.

17. Hirasaki S, Suzuki S, Umemura S, Kamei H, Okuda M and Kudo K: Asymptomatic colonic metastases from primary squamous cell carcinoma of the lung with a positive fecal occult blood test. World J Gastroenterol 14: 5481-5483, 2008.

18. Habeşoğlu MA, Oğuzülgen KI, Oztürk C, Akyürek N and Memiş L: A case of bronchogenic carcinoma presenting with acute abdomen. Tuberk Toraks 53: 280-283, 2005.

19. Carroll D and Rajesh PB: Colonic metastases from primary squamous cell carcinoma of the lung. Eur J Cardiothorac Surg 19: 719-720, 2001.

20. Sakai H, Egi H, Hinoi T, Tokunaga M, Kawaguchi Y, Shinomura M, Adachi T, Arihiro K and Ohdan H: Primary lung cancer presenting with metastasis to the colon: A case report. World J Surg Oncol 10: 127, 2012.

21. Stinchcombe TE, Socinski MA, Gangarosa LM and Khandani AH: Lung cancer presenting with a solitary colon metastasis detected on positron emission tomography scan. J Clin Oncol 24: 4939-4940, 2006. 
22. Yang CJ, Hwang JJ, Kang WY, Chong IW, Wang TH, Sheu CC, Tsai JR and Huang MS: Gastro-intestinal metastasis of primary lung carcinoma: Clinical presentations and outcome. Lung Cancer 54: 319-323, 2006.

23. Jia J, Ren J, Gu J, Di L and Song G: Predominant sarcomatoid carcinoma of the lung concurrent with jejunal metastasis and leukocytosis. Rare Tumors 2: e44, 2010.

24. Byers TE, Vena JE and Rzepka TF: Predilection of lung cancer for the upper lobes: An epidemiologic inquiry. J Natl Cancer Inst 72: 1271-1275, 1984.

25. Coleman MP, Babb P, Mayer D, Quinn MJ and Sloggett A: Cancer survival trends in England and Wales, 1971-1995: Deprivation and NHS Region. London: Office for National Statistics, 1999.

26. Casella G, Di Bella C, Cambareri AR, Buda CA, Corti G, Magri F Crippa S and Baldini V: Gastric metastasis by lung small cell carcinoma. World J Gastroenterol 12: 4096-4097, 2006.

27. Suzaki N, Hiraki A, Ueoka H, Aoe M, Takigawa N, Kishino T, Kiura K, Kanehiro A, Tanimoto M and Harada M: Gastric perforation due to metastasis from adenocarcinomaof the lung. Anticancer Res 22: 1209-1212, 2002.

28. Yamamoto M, Matsuzaki K, Kusumoto H, Uchida H, Mine H, Kabashima A, Maehara Y and Sugimachi K: Gastric metastasis from lung carcinoma. Case report. Hepatogastroenterology 49: 363-365, 2002.

29. Garwood RA, Sawyer MD, Ledesma EJ, Foley E and Claridge JA: A case and review of bowel perforation secondary to metastatic lung cancer. Am Surg 71: 110-116, 2005.
30. Sakorafas GH, Pavlakis G and Grigoriadis KD: Small bowel perforation secondary to metastatic lung cancer: A case report and review of the literature. Mt Sinai J Med 70: 130-132, 2003.

31. Atsushi G, Tsutomu K, Takao T, Hidenori K, Masayuki K and Kiyoshi S: A case report of colon metastasis from lung cancer with a long survival time. J Jpn Associat Chest Surg 26: 515-519, 2012 (In Japanese).

32. Inoshita T, Youngberg GA and Thur De Koos P: Esophageal metastasis from a peripheral lung carcinoma masquerading as a primary esophageal tumor. J Surg Oncol 24: 49-52, 1983.

33. Hsu PK, Shai SE, Wang J and Hsu CP: Esophageal metastasis from occult lung cancer. J Chin Med Assoc 73: 327-330, 2010.

34. Yoshino I, Yohena T, Kitajima M, Ushijima C, Nishioka K, Ichinose Y and Sugimachi K: Survival of non-small cell lung cancer patients with postoperative recurrence at distant organs. Ann Thorac Cardiovasc Surg 7: 204-209, 2001.

35. Lardinois D, Weder W, Roudas M, von Schulthess GK, Tutic M, Moch H, Stahel RA and Steinert HC: Etiology of solitary extrapulmonary positron emission tomography and computed tomography findings in patients with lung cancer. J Clin Oncol 23: 6846-6853, 2005.

36. Shiono S, Masaoka T, Sato T and Yanagawa N: Positron emission tomography (PET)-computed tomography (CT) suggesting small intestinal metastasis from lung cancer; report of a case. Kyobu Geka 59: 426-429, 2006 (In Japanese). 